\title{
Full-scale Improved Delayed Detached Eddy Simulation of Transverse Hydrogen Jet in Supersonic Combustion
}

\author{
E Fan $^{1 \mathrm{a}}$ Kun Wu ${ }^{2 \mathrm{a}}$ Yachao Lee ${ }^{3 \mathrm{a}}$ Wei $\mathrm{Yao}^{4 \mathrm{a}, \mathrm{b}}$ and Xuejun Fan ${ }^{5 \mathrm{a}, \mathrm{b}}$
}

a. Key Laboratory of High Temperature Gas Dynamics, Institute of Mechanics, CAS, No.15 Beisihuanxi Road, Beijing 100190, China

b. School of Engineering Science, University of Chinese Academy of Science, Beijing 100049, China

Realizing highly efficient supersonic combustion is critical for the development of scramjets. The ground test data of scramjet combustors are both expensive and difficult to be measured, thus high-fidelity numerical simulation becomes a necessary way for supersonic combustion research. By aid of the in-house developed compressible reacting flow solver AstroFoam, the hybrid Reynolds-Averaged Navier-Stokes/Large Eddy Simulation (RANS/LES) turbulence modeling framework based on Improved Delayed Detached Eddy Simulation (IDDES) and the Partially Stirred Reactor (PaSR) turbulent combustion model are used for the study of HyShot II scramjet tested in the high enthalpy shock tunnel in Göttingen (HEG). A detailed mechanism of $\mathrm{H}_{2}$ /Air combustion with 9 species and 19 elementary reactions is used. The predicted static pressure distribution agrees well with experimental data. Typical flow structures of jet in supersonic cross flow including the evolvement of $S$-shaped structure to $\Omega$-shaped structure are captured. Based on the distribution of temperature and $\mathrm{OH}$ mass fraction, the flow field can be divided into three zones: the mixing zone, the ignition zone and the turbulent combustion zone. Analysis of the cross-sections in the ignition zone along the streamwise direction reveals that the initial emergence $\mathrm{OH}$ reactant lies mainly in the shear layer, and the Kelvin-Helmholtz instability is the underlying stimulation of the ignition.

\section{Nomenclature}

$C_{D E S}=$ length coefficient for LES branch in IDDES model

$C_{\text {mix }}=$ coefficient for characteristic time of mixing process

$C_{w} \quad=$ length coefficient for distance to wall

$d_{w} \quad=$ distance to wall in IDDES model

$d_{\text {wall }}=$ near wall distance in original S-A model

$D=$ diameter of fuel nozzle

$h_{\max }=$ grid largest length scale

$h_{w n} \quad=$ grid length scale in wall-normal direction

$S_{i j} \quad=$ strain rate

$\varepsilon=$ dissipation rate of turbulent kinetic energy

$\phi \quad=$ equivalence ratio

$\tau_{c} \quad=$ characteristic time of chemistry

$\tau_{\text {mix }}=$ characteristic time of mixing process

$\tau_{c}=$ characteristic time of turbulence

$\omega_{l} \quad=$ turbulent reaction rate

$\omega_{t} \quad=$ turbulent reaction rate

$\Delta_{\text {grid }}=$ subgrid length scale

\footnotetext{
Master candidate, Institute of Mechanics (CAS), fane@imech.ac.cn, AIAA member.

${ }^{2} \mathrm{PhD}$ Candidate, Institute of Mechanics (CAS), wukun@imech.ac.cn, AIAA member.

${ }^{3} \mathrm{PhD}$ Candidate, Institute of Mechanics (CAS), liyachao@imech.ac.cn, AIAA member.

${ }^{4}$ Associate Professor, Institute of Mechanics (CAS), weiyao@imech.ac.cn, AIAA member, corresponding author.

${ }^{5}$ Professor, Institute of Mechanics (CAS), xfan@imech.ac.cn, AIAA lifetime member, corresponding author.
} 


\section{Introduction}

$\mathrm{H}^{\prime \prime}$ alf century of scramjet study has made considerable achievement in the illumination of the mixing and combustion mechanism of supersonic combustion. However, due to the deficiency of effective measurement techniques, neither flight nor ground tests have revealed the most profound physics in scramjet combustor. Here, a numerical study of HyShot II scramjet based on detailed $\mathrm{H}_{2}$ /Air combustion mechanism is made to help understand the aerothermochemistry in supersonic combustion with transverse fuel jet.

Jets in supersonic crossflow (JISC) is a canonical problem in the supersonic combustion studies especially in transverse jet, as the complex three-dimensional (3-D) flow structures significantly influence the fuel/air mixing and the subsequent self-ignition process. Early studies of JISC mostly focused on the mixing mechanism. Experimental studies $[1,2]$ observed the analogy in vortex structures and pressure distribution between the transverse jet and the cylinder disturbance in a supersonic flow, and thus proposed that jet penetration can be treated as an equivalent obstacle. Chenault et al. [3] investigated the effect of different turbulence models on the modeling of jet disturbance, and found that Reynold's Stress Model (RSM) accurately predicted time-averaged characteristics and main flow structures of JISC, as well as captured the second flow. The large eddy simulation (LES) simulation of JISC with Mach 1.6 free-stream by Rana et al. [4] demonstrated that Kelvin-Helmholtz type instabilities were dominantly responsible for the mixing process.

Study of JISC has a practical meaning for the design scramjet combustors as the fuel is typically injected perpendicularly to supersonic free-stream in most combustors. In order to avoid the uncertainties in modeling complex hydrocarbon chemistry and focus on the impact of JISC on supersonic combustion, a $\mathrm{H}_{2}$ fueled scramjet combustor is selected for numerical study rather than ethylene or kerosene. Pioneering flight test of HyShot II [5] scramjet motivated many later experimental and numerical studies of supersonic combustion, including the ground test of HyShot II in High Enthalpy Shock Tunnel Göttingen (HEG) of Deutsches Zentrum für Luft-und Raumfahrt (DLR) [5]. In this study, the mixing and combustion process of HyShot II in the HEG ground test were reproduced and analyzed by Improved Delayed Detached Eddy Simulation (IDDES) [6] with Partially Stirred Reactor (PaSR) [7]. A detailed mechanism of $\mathrm{H}_{2}$ /Air combustion with 9 species and 19 elementary reactions is used to simulate the combustion chemistry.

\section{HyShot II flight test and HEG ground based experiment}

HyShot II flight test took place in central Australia. A two-stage Terrier-Orion Mk70 rocket is used to achieve an apogee in excess of $300 \mathrm{~km}$ with a highly parabolic trajectory. The combination of rocket and trajectory allowed the test-section to reenter the atmosphere with a Mach number higher than 7.5 and between 35 and $25 \mathrm{~km}$ altitude. Postflight analysis was performed in HEG utilizing 1:1 scale wind tunnel. Results showed qualitative agreement with the flight data and confirmed the establishment of supersonic combustion. In this study we consider HEG experiment condition XII, which corresponds to the flight condition at an altitude of $32.5 \mathrm{~km}$ and the global equivalence ratio is $\phi=0.43$. The flow-path of the HEG shock tunnel duplicates the flight configuration and consists of a intake ramp, a rectilinear combustion chamber and a single-sided exhaust nozzle, as shown in Figure 1. The intake ramp has a halfangle of $18^{\circ}$. The bleed channels have a length of $5 \mathrm{~mm}$, while the combustion chamber has a length of $300 \mathrm{~mm}$ and a cross-section of $9.8 \times 75.0 \mathrm{~mm}^{2}$. Gaseous $\mathrm{H}_{2}$ at sonic speed is injected perpendicularly to the air stream through 4 identical flush-mounted injectors with a diameter of $\mathrm{D}=2.0 \mathrm{~mm}$, which lie at $58 \mathrm{~mm}$ downstream of the leading edge of the combustor. Fourteen pressure transducers were used for the static pressure measurement. Compared to the flight test, the HEG facilities provide more controllability of the test conditions and more detailed measurements, which is an ideal CFD modeling target.

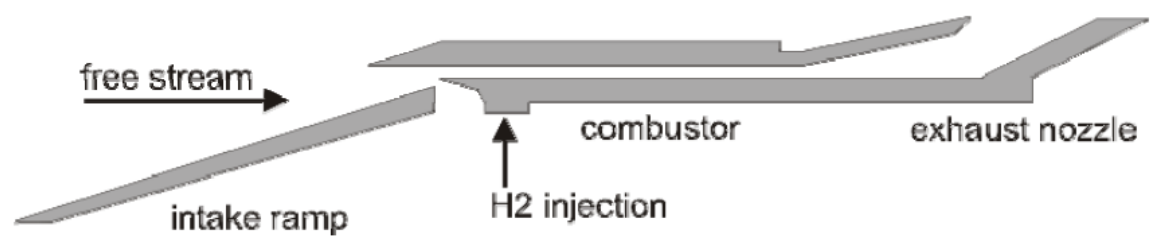

Figure 1 Schematic of the HyShot II scramjet configuration

\section{HEG computational set-up}

The HyShot II is composed of intake ramp, combustor and exhaust nozzle as show in Figure 1. Since side and floor bleeds are used to spill the boundary layers from the flow, the combustor can be treated as separated from the 
inlet nozzle, thus the study will only model the combustor and the exhaust nozzle for simplicity. At the combustor inlet, the boundary conditions for pressure, velocity and temperature in transverse direction are taken from the RANS modeling of the inlet nozzle by Schramm et al. [8]. Gaseous $\mathrm{H}_{2}$ is injected perpendicularly to free stream at four fuel injectors located $58 \mathrm{~mm}$ downstream of the chamber leading edge. To alleviate the computational burden, only $1 / 4$ of the flow-path is modeled by using the combustor symmetries, and symmetry boundary condition is applied to splitting planes. The wall temperature is fixed at $300 \mathrm{~K}$ assuming that the wall temperature rise is small during the short test duration. A sonic nozzle with an inlet diameter of 4D is designed for the fuel injection. Details of the boundary conditions are shown in Table. 1. Two sets of mesh as shown in Figure 2 are used in this study, including a structured coarse mesh with 5.2 million cells and a refined mesh with 18.5 million cells. All the results in Part V are based on the refined mesh (referred to as Mod-refined) unless otherwise indicated.

Table 1 Boundary Conditions for the HEG Simulation

\begin{tabular}{|c|c|c|c|c|}
\hline & $\mathrm{P}\left(10^{5} \mathrm{~Pa}\right)$ & $\mathrm{T}(\mathrm{K})$ & $\mathrm{U}(\mathrm{m} / \mathrm{s})$ & Mass Fraction \\
\hline Combustor inlet & \multicolumn{2}{|c|}{ Adopted from RANS modeling [5], Dirichlet } & $\begin{array}{l}\mathrm{O}_{2}: 0.233 \\
\mathrm{~N}_{2}: 0.767 \\
\mathrm{H}_{2}: 0\end{array}$ \\
\hline Fuel nozzle inlet & $\begin{array}{c}2.97 \\
\text { (Total } \\
\text { pressure })\end{array}$ & 300 & 0 & $\mathrm{H}_{2}: 1$ \\
\hline $\begin{array}{c}\text { Walls of combustor, } \\
\text { expander and fuel } \\
\text { nozzle }\end{array}$ & Zero gradient & 300 & 0 & Zero gradient \\
\hline $\begin{array}{c}\text { Combustor outlet } \\
\text { Side walls for } \\
\text { combustor }\end{array}$ & \multicolumn{4}{|c|}{$\begin{array}{c}\text { Zero gradient, } \\
\text { supersonic outlet }\end{array}$} \\
\hline \hline
\end{tabular}

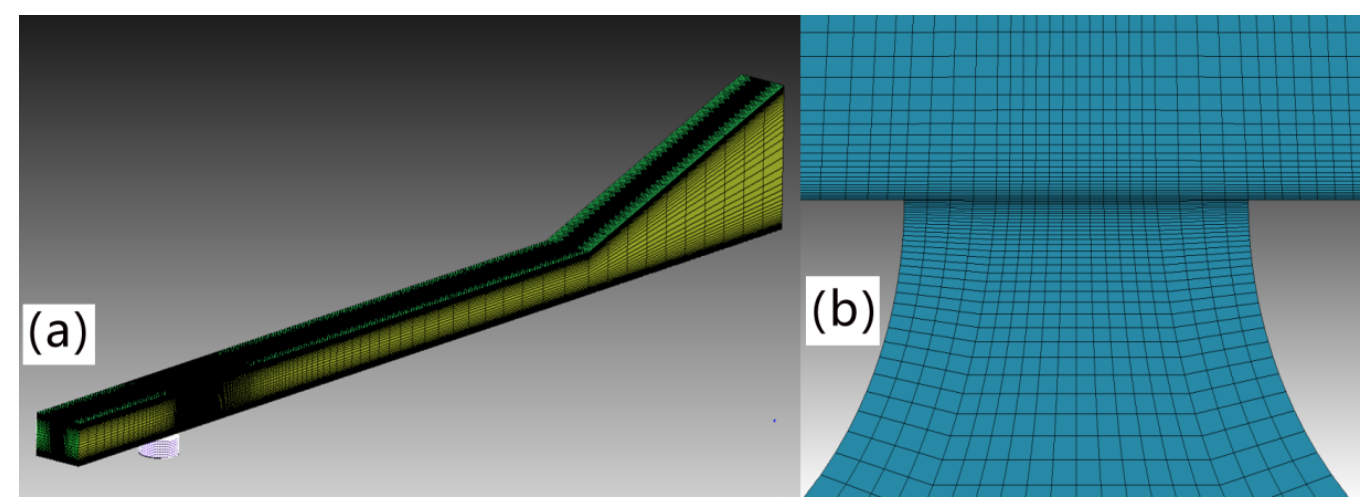

Figure 2 CFD mesh for the HEG chamber: (a) global view of the structured mesh with 5.2 million cells, (b) local view of the $\mathrm{H}_{2}$ fuel nozzle cut at the middle plane

\section{Mathematical models and numerical methods}

IDDES approach is used for the turbulence modeling to reduce the computational cost in boundary layer modeling as well as capture the instantaneous flow structures. IDDES is a hybrid Reynolds Average Navier Stokes / Large Eddy Simulation (RANS/LES) approach for the turbulence modeling, RANS is applied in near-wall regions to avoid the huge cell number required for LES, and LES is applied in regions far from the wall to accurately describe the instantaneous processes of mixing and combustion. The one-equation Spalart-Allmaras (S-A) model is used as the background RANS model to cover the boundary layer through well-designed inflation grid layer. The SA model was designed for wall-bounded flows in aerospace applications, and shown good performance in boundary layer separating prediction [9]. Since S-A model only adds one extra unknown variable knows as modified turbulent kinetic viscosity, it's relatively simple and computationally efficient. The IDDES method is an advanced version of DES97 and DDES, with progressive and well-designed subgrid length scale to avoid premature separation and 
mismatched log-layer. In the original S-A model near wall distance $\mathrm{d}_{\text {wall }}$ is used as length scale, while in IDDES the subgrid length scale $\Delta_{\text {grid }}$ is used instead [6]

$$
\Delta_{\text {grid }}=\min \left\{\max \left[\mathrm{C}_{\mathrm{w}} \mathrm{d}_{\mathrm{w}}, \mathrm{C}_{\mathrm{w}} \mathrm{h}_{\text {max }}, \mathrm{h}_{\mathrm{wn}}\right], \mathrm{h}_{\max }\right\}
$$

where $d_{w}$ is distance to wall, $h_{\max }$ is the grid largest length scale in flow direction, wall-normal direction and spanwise direction, $h_{w n}$ is the grid length scale in wall-perpendicular direction and the coefficient $C_{w}=0.15$. The LES model is also closed by the $\mathrm{S}$-A one-equation model through setting the length scale as $\mathrm{C}_{\mathrm{DES}} \Delta_{\text {grid }}$ in the LES region, where $\mathrm{C}_{\mathrm{DES}}=0.65$.

Since the characteristic time scales of chemistry $\tau_{c}$ and turbulence $\tau_{t}$ share a close magnitude in supersonic combustion, PaSR model based on finite-rate chemistry model is used for the turbulent combustion modeling. Golovitchev et. al [7] proposed a PaSR model considering the effect of imperfection mixing on turbulent reaction rate through defining the turbulent reaction rate $\omega_{t}$ by

$$
\omega_{\mathrm{t}}=\omega_{1} \frac{\tau_{\mathrm{c}}}{\tau_{\mathrm{c}}+\tau_{\text {mix }}}
$$

where $\omega_{l}$ is laminar reaction rate. $\tau_{\mathrm{mix}}$ is the characteristic time of mixing process and determined by

$$
\tau_{\text {mix }}=\mathrm{C}_{\text {mix }}\left(\frac{v_{\text {eff }}}{\varepsilon}\right)^{1 / 2}
$$

where $\mathrm{C}_{\text {mix }}=1, \varepsilon$ is the dissipation of turbulent kinetic energy and is calculated as $\varepsilon=2 v_{\text {eff }}\left|\mathrm{S}_{\mathrm{ij}}\right|^{2}$ based on S-A model with $S_{i j}$ is strain rate. Based on these equations, turbulent reaction rate is controlled by rate of imperfect mixing zone converting to perfect mixing zone.

The $\mathrm{H}_{2}$ /Air combustion mechanism from Burke et. al [10] is selected for it is sensitive to the pressure variation. After removing argon and helium participating reactions, a skeletal mechanism with 9 species and 19 elementary reactions is used as the chemistry input.

The modeling in this study employed an FVM (Finite Volume Method) based on in-housed developed compressible reacting flow solver AstroFOAM, which was extended from the compressible non-reacting solver rhoCentralFOAM distributed with OpenFOAM package mainly through adding the features of multi-species transport and multi-component reaction. The inviscid convective fluxes were evaluated by central KurganovTadmor (KT) scheme, which assumes a low numerical dissipation in resolving discontinuities (e.g. shock) yet a high computational efficiency due to its Riemann-free simplicity. TVD (Total Variation Diminishing) scheme at cell face with Minimod limiter is used to achieve second-order spatial accuracy. Mixture is assumed to be ideal gases in thermal equilibrium. Thermodynamic curve fits from McBride et al. [11] are used for species-specific heats and enthalpies. Molecular viscosity is calculated based on Sutherland formula, then constant Prandtl number and Schmidt number with the value of 1.0 are applied for the calculation of turbulent thermal and mass diffusion coefficients. Direct integration (DI) method is used for the stiff chemistry system solving.

\section{Results and discussion}

Figure 3 compares the predicted time-averaged and measured [5] wall pressure distribution, along with the LES result from Fureby et. al [12]. The predictions by the coarse mesh and the refined mesh are generally close to each other except for those between $\mathrm{x}=0.05 \mathrm{~m}$ and $\mathrm{x}=0.2 \mathrm{~m}$, where the mesh refinement is mainly located in this region. The agreement with experimental data is good, except for small deviations between $\mathrm{x}=0.05 \mathrm{~m}$ and $\mathrm{x}=0.2$ $\mathrm{m}$. The discrepancies between the two mesh sets and the deviations against the measurements all occur in this regions, which locate in a short distance downstream of the injection and correspond the main vortex generation regions. Thus it implies that a high mesh resolution may be critical to accurately capture the flow patterns there. Both the current and Fureby's results well predict the general pressure profile, and the current refined modeling results show better agreement with the measurements in the latter part of the burner section from $\mathrm{x}=0.17 \mathrm{~m}$ to $\mathrm{x}=$ 0.3 . 


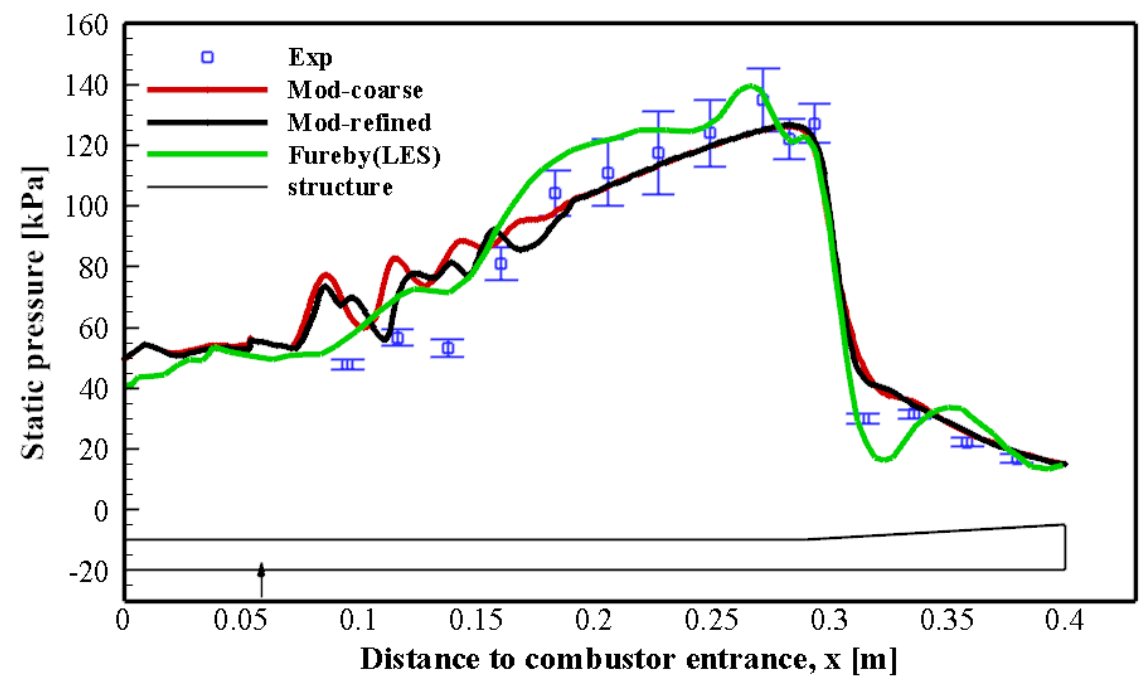

Figure 3 Comparison of predicted time averaged and measured wall pressure distribution, the arrow indicates location of fuel injector.

Figure 4 shows the contours of instantaneous static pressure, temperature, Mach number, $\mathrm{H}_{2}$ mass fraction on the middle streamwise plane. Bow Shock and shock trains can be detected in the pressure contour. Pressure rises in the latter half of the burner section due to volumetric expansion caused by combustion and decreases in the expander section as a consequence of increasing cross-section area. The high temperature $(>2000 \mathrm{~K})$ zone mainly locates in the latter half of the burner and the entrance of the expander due to the intense heat release from the $\mathrm{H}_{2} / \mathrm{Air}$ combustion. The Mach number contour shows a continuous supersonic flow in the burner section, implying that the combustor is running at scramjet mode. The low temperature $(<600 \mathrm{~K})$ zone located immediately following the jet injection and near the wall corresponds to the cold $\mathrm{H}_{2}$ jet wake. The flow field is mostly in supersonic status and isolated subsonic regions lie mainly around the fuel nozzle. The jet penetration identified from the $\mathrm{H}_{2}$ mass fraction contour is about 2D. The mixing distance marked by the fully dispersion of $\mathrm{H}_{2}$ is around 20D.

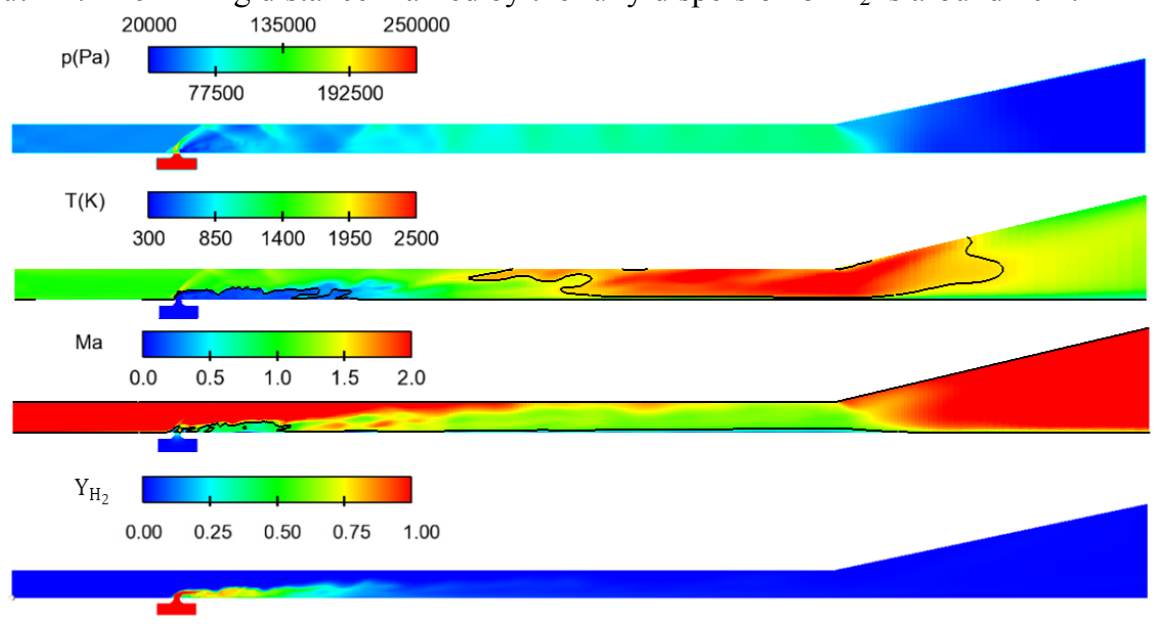

Figure 4 Instantaneous static pressure, temperature, Mach number, $\mathbf{H}_{2}$ mass fraction contours at the midline transverse plane at $2.543 \mathrm{~ms}$, with isoline of $600 \mathrm{~K}$ and $2000 \mathrm{~K}$ in temperature contour and sonic line in Mach number contour

Figure 5 shows a close-up view of the two-dimensional (2-D) JISC flow structures on the middle plane, which are widely observed in both experimental and numerical studies. As seen, typical features are well captured by refined mesh. The boundary layer separates immediately upstream of the fuel nozzle, which leads to the $\lambda$-shape shock, the fuel jet acts as an obstacle and forces the supersonic main flow to circumvent it and form the bow shock. Two recirculation zones, named R1 and R2 are formed between the $\lambda$ shock and the bow shock, and between the bow shock and the fuel jet respectively. The supersonic fuel jet shows typical features of under-expanded jets, including the Mach barrel, the Prandtl-Meyer expansion fan, the Mach disk, reflected shocks and triple points. It's 
observed that the maximum Mach number lies at the top end of the jet plume just before the Mach disk. A new recirculation zone named $\mathrm{R} 3$ is produced at the downstream of the fuel jet.

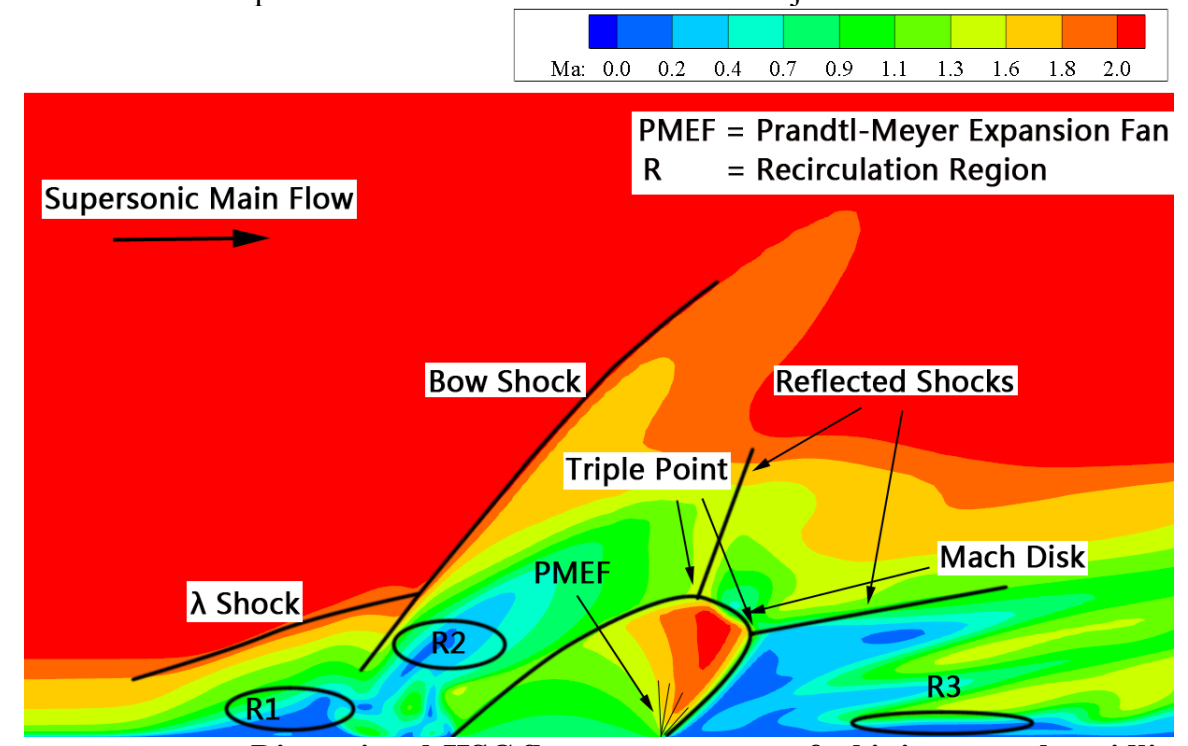

Figure 5 Instantaneous two Dimensional JISC flow structure near fuel injector at the midline transverse plane at $2.543 \mathrm{~ms}$

Figure 6 shows the three-dimensional (3-D) iso-surface of the Q-criterion (the second invariant of the velocity gradient tensor) colored by $\mathrm{H}_{2}$ mass fraction. Typical vortex structures of JISC, e.g. the horseshoe vortex, the counter rotating vortices (CRVs) and finer vortices in complex structures can be detected. These structures include S-shaped vortices with long arms stretching in the streamwise direction and align with the jet wake, and tips curling over the jet plume forming parcels. Although the jet core as it mixes with the main flow, the difference of streamwise velocity between the jet plume and the main stream leads to the shear layer. The S-shaped vortices have also been observed by Ben-Yakar et. al. [13] in their experiment, and they proposed that the stretch movement of the long arms is enhanced by the shear stresses in the shear layer. These structures seem to originate from the Kelvin-Helmholz instability in the shear layer. Further downstream, the tips of different S-shaped vortices merge, and adjacent $\mathrm{S}$-shaped vortices coalesce into $\Omega$-shaped vortices. The $\Omega$-shaped vortices break down into CRVs as they travel downstream.

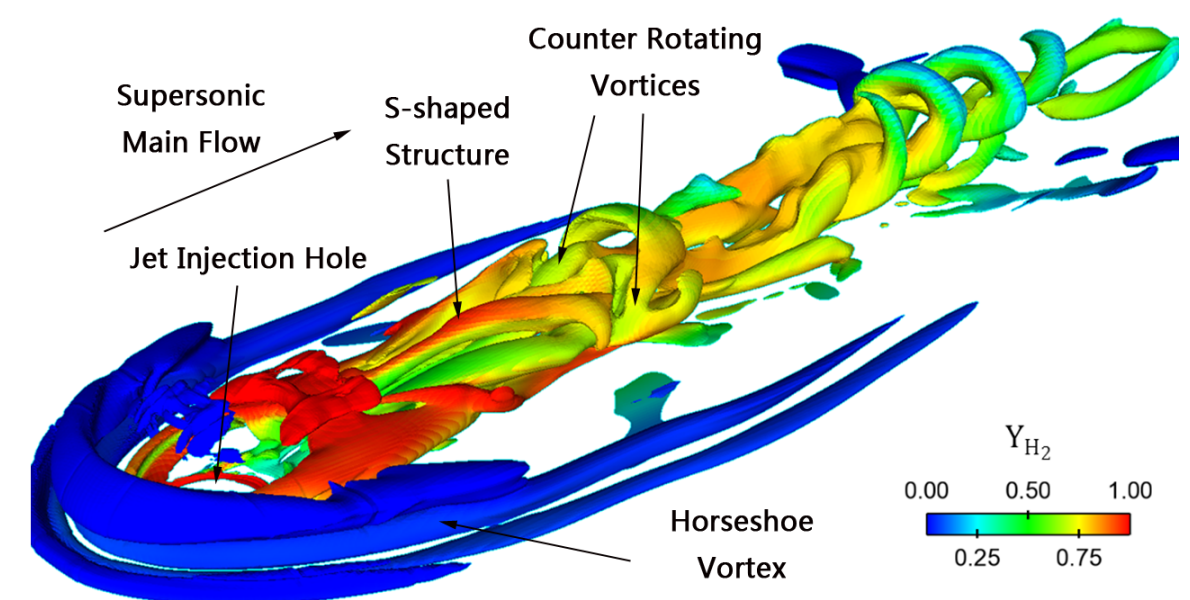

Figure 6 Instantaneous three-dimensional iso-surface for the Q-criterion, colored by $\mathrm{H}_{2}$ mass fraction at 2.543 ms

Figure 7 shows the 3-D iso-surfaces of the Q-criterion colored by $\mathrm{OH}$ mass fraction and temperature respectively. According to the distribution of $\mathrm{OH}$ mass fraction and temperature, the flow field can be generally divided into three zones: the mixing zone, the ignition zone and the turbulent combustion zone. In the mixing zone, which lies about 20D downstream of fuel nozzle, the combustion is too weak to produce apparent $\mathrm{OH}$ reactants, and the

6

American Institute of Aeronautics and Astronautics 
mixing process dominates in this zone and prepares for the downstream ignition. Further downstream in the ignition zone, the fuel and the air are well mixed, remarkable $\mathrm{OH}$ arises but the temperature is still low $(<2000 \mathrm{~K})$ there. As the flow approaching to the end of the combustor, it turns to be fully developed turbulent flow and both $\mathrm{OH}$ mass fraction and temperature reach their peaks within this zone. It's not surprising to find that the turbulent combustion zone coincides with the high temperature $(>2000 \mathrm{~K})$ zone shown in Figure 4.

Figure 8 (a) shows instantaneous temperature and $\mathrm{OH}$ mass fraction contours at different cross-sections. Most of the contours show highly symmetric except the fourth one. The first cross-section locates in the mixing zone and the low temperature below $600 \mathrm{~K}$ near the bottom wall is caused by colder jet plume. Later in the second crosssection the low-temperature region is separated from the wall as the jet core lifting up. Further downstream in the third cross-section, huge amounts of $\mathrm{OH}$ reactants is detected and the peak temperature reaches about $2000 \mathrm{~K}$. Later cross-sections all locate in the turbulent combustion zone, where the increases in both temperature and $\mathrm{OH}$ mass fraction imply intense combustion reactions there. Figure 8(b) shows the contour of temperature and some key reactants superposed with the streamline in the second cross-section. CRVs can be clearly identified from the streamline, which coincides with the jet core marked by high concentration of $\mathrm{H}_{2}$. Since the consumption of $\mathrm{O}_{2}$ and production of $\mathrm{OH}$ are mostly spotted in the shear layer, which lies between the jet core and the main flow around the CRVs, the Kelvin-Helmholtz instability could be the underlying stimulation of the ignition.

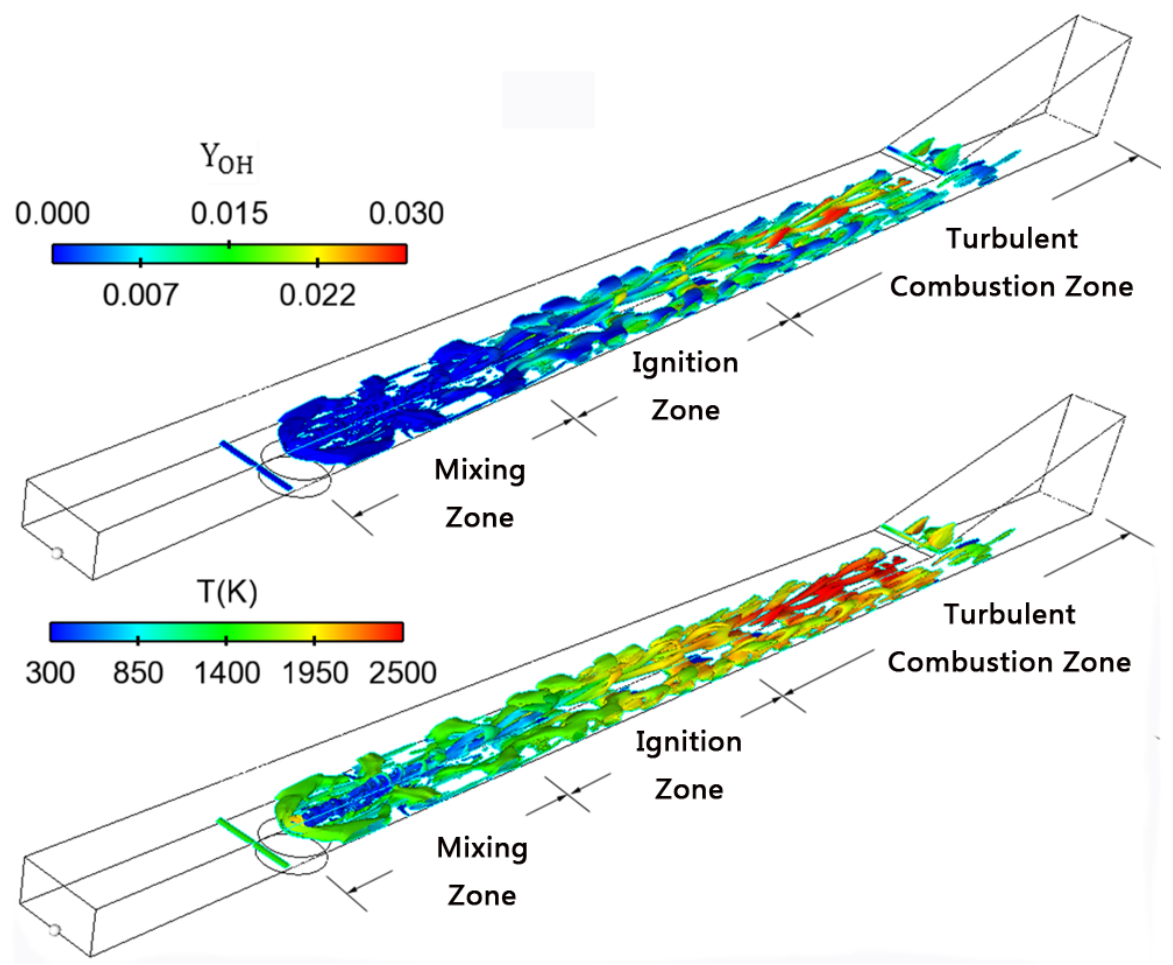

Figure 7 Overall view of instantaneous three-dimensional iso-surface for the Q-criterion, colored by $\mathrm{OH}$ mass fraction and temperature at $2.543 \mathrm{~ms}$ 


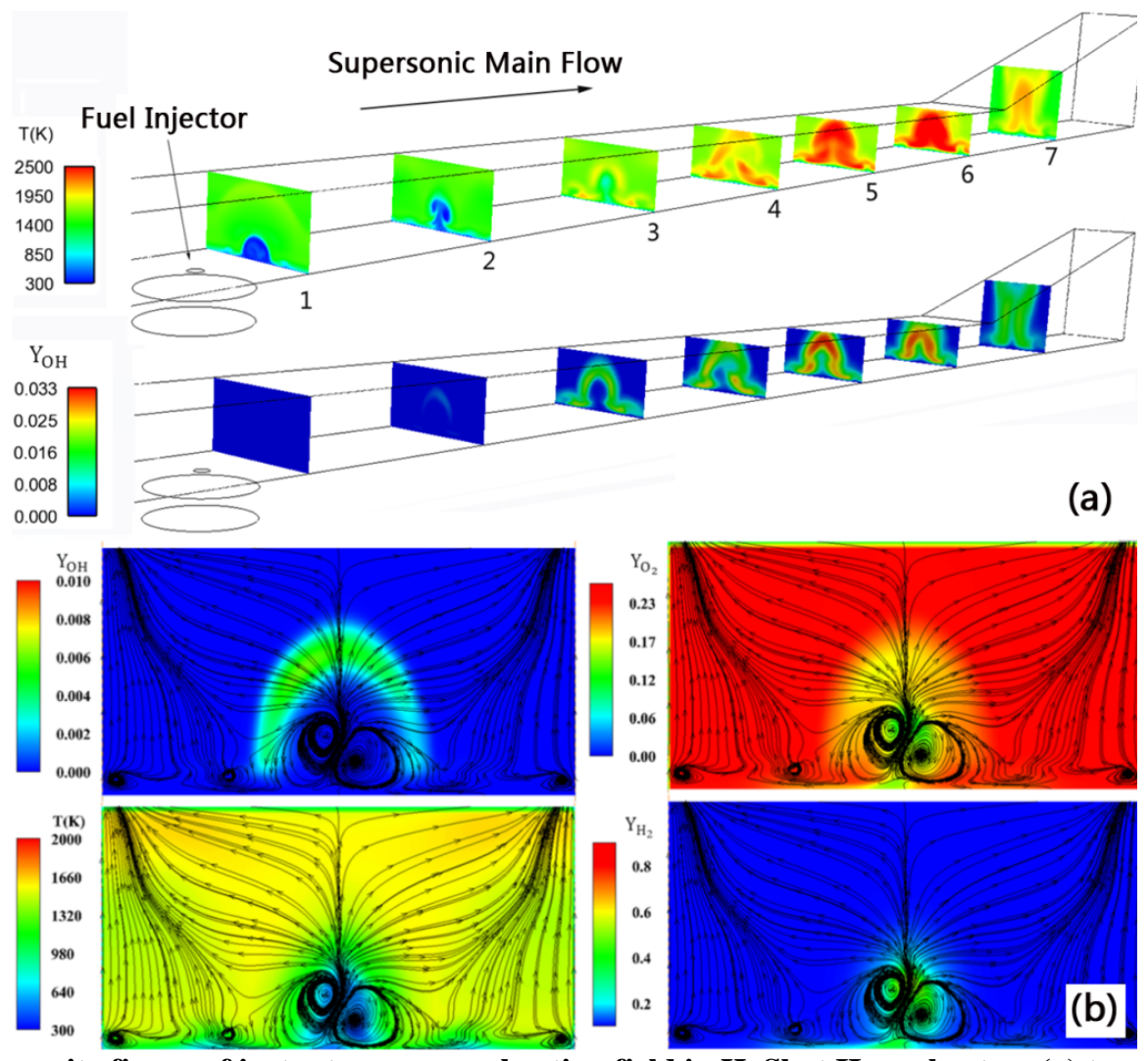

Figure 8 Composite figure of instantaneous combustion field in HyShot II combustor: (a) temperature and $\mathrm{OH}$ mass fraction contour at different streamwise cross-section s; (b) temperature and mass fraction of $\mathrm{OH}$, $\mathrm{O}_{2}, \mathrm{H}_{2}$ contour superposed with streamline at second cross-section

\section{Conclusion}

In this study, IDDES coupled with PaSR is employed to analyze the mixing and combustion characteristics of supersonic combustion based on the HEG ground tests of HyShot II scramjet, which corresponds to the flight conditions at an altitude of $32.5 \mathrm{~km}$. The predicted pressure distribution agrees well with the experimental data, especially in the latter part of the burner section. Typical flow structures of JISC are captured, and the evolvement of S-shaped structure to $\Omega$-shaped structure is also detected. These complicated pattern vortices are believe to enhance mixing and further ignition process. Based on the distribution of temperature and $\mathrm{OH}$ mass fraction, the flow field is divided into three zones: the mixing zone, the ignition zone and the turbulent combustion zone. Analysis of reactants and temperature distribution on cross-sections in the ignition zone reveals that the initial emergence $\mathrm{OH}$ reactant lies mainly in the shear layer, and the Kelvin-Helmholtz instability could be the underlying stimulation of the ignition, which merits further studies.

\section{Acknowledgments}

The work was supported by National Natural Science Foundation of China (Grant No. 11502270) and Training Program of Major Research Plan of the National Natural Science Foundation of China (Grant No. 91641110). The authors are also grateful to National Supercomputer Centre in Tianjin for providing computational resource.

\section{References}

1. Glagolev, A., Zubkov, A., and Panov, Y. A. "Supersonic flow past a gaseous jet obstacle on a plate," Izv. Akad. Nauk SSSR, Mekh. Zhidk. Gaza, No. 3, 1967, p. 97.

2. Glagolev, A., Zubkov, A., and Panov, Y. A. "Interaction between a supersonic flow and gas issuing from a hole in a plate," Fluid Dynamics Vol. 3, No. 2, 1968, pp. 65-67. 
3. Chenault, C. F., Beran, P. S., and W. Bowersox, R. D. "Numerical investigation of supersonic injection using a Reynolds-stress turbulence model," AIAA journal Vol. 37, No. 10, 1999, pp. 1257-1269.

4. Rana, Z. A., Thornber, B., and Drikakis, D. "Transverse jet injection into a supersonic turbulent cross-flow," Physics of Fluids Vol. 23, No. 4, 2011, p. 046103.

5. Schramm, J. M., Karl, S., Hannemann, K., and Steelant, J. "Ground testing of the HyShot II scramjet configuration in HEG," AIAA Paper Vol. 2547, 2008, p. 2008.

6. Shur, M. L., Spalart, P. R., Strelets, M. K., and Travin, A. K. "A hybrid RANS-LES approach with delayed-DES and wall-modelled LES capabilities," International Journal of Heat and Fluid Flow Vol. 29, No. 6, 2008, pp. 1638-1649.

7. Golovitchev, V., and Chomiak, J. "Numerical Modeling of High-Temperature Air Flameless Combustion," The 4th international symposium on high temperature air combustion and gasification. Rome. Italy. 2001.

8. Smart, M. K., Hass, N. E., and Paull, A. "Flight data analysis of the HyShot 2 scramjet flight experiment," AIAA journal Vol. 44, No. 10, 2006, pp. 2366-2375.

9. $\quad$ Spalart, P. R., and Allmaras, S. R. "A one equation turbulence model for aerodinamic flows," AIAA journal Vol. 94, 1992.

10. Burke, M. P., Chaos, M., Ju, Y., Dryer, F. L., and Klippenstein, S. J. "Comprehensive H2/O2 kinetic model for high pressure combustion," International Journal of Chemical Kinetics Vol. 44, No. 7, 2012, pp. 444-474.

11. Mcbride, B. J., Gordon, S., and Reno, M. A. "Coefficiennts for calcullating thermodynamics and transport properties of individual species." National Aeronautics and Space Administration, 1993.

12. Fureby, C., Chapuis, M., Fedina, E., and Karl, S. "CFD analysis of the HyShot II scramjet combustor," Proceedings of the Combustion Institute Vol. 33, No. 2, 2011, pp. 2399-2405.

13. Ben-Yakar, A., Mungal, M., and Hanson, R. "Time evolution and mixing characteristics of hydrogen and ethylene transverse jets in supersonic crossflows," Physics of Fluids Vol. 18, No. 2, 2006, p. 026101. 